Original Paper

\title{
Internal Flow and Limiting Streamlines Observations of Contra-Rotating Axial Flow Pump at Partial Flow Rate
}

\author{
Satoshi Watanabe ${ }^{1}$, Shimpei Momosaki ${ }^{2}$, Satoshi Usami ${ }^{2}$ and Akinori Furukawa ${ }^{1}$ \\ ${ }^{1}$ Department of Mechanical Engineering, Kyushu University \\ 744 Motooka, Nishi-ku, Fukuoka 819-03965, Japan, \\ fmnabe@mech.kyushu-u.ac.jp, fmfuru@mech.kyushu-u.ac.jp \\ ${ }^{2}$ Graduate School of Engineering, Kyushu University \\ 744 Motooka, Nishi-ku, Fukuoka 819-03965, Japan, \\ s.momosaki@fcs.mech.kyushu-u.ac.jp, usami@ryusei2.mech.kyushu-u.ac.jp
}

\begin{abstract}
An application of contra-rotating rotors, in which a rear rotor is in tandem with a front one and these rotors rotate in the opposite direction each other, has been proposed against a demand for developing higher specific speed axial flow pump. One prototype rotors, which we have designed with a conventional method, has given the positive slope of head characteristic curve especially in the rear rotor. It is necessary to understand the internal flow behavior in the rear rotor to establish the design guideline for achieving higher and more reliable performance. In the present study, we carried out the experimental investigations of the internal flow field of the rear rotor, especially at the partial flow rate, by Laser Doppler Velocimetry (LDV) for the main flow and the limiting streamlines observation on rotor surfaces for the boundary layer flows.
\end{abstract}

Keywords: Axial flow pump, Contra-rotating rotors, Partial flow rate, LDV, Limiting streamlines

\section{Introduction}

Recently, there is a strong demand for developing higher specific speed axial flow pump in reduction of pump installation space and costs [1, 2]. However, the conventional axial flow pump with higher specific speed suffers from the deterioration of efficiency and cavitation performance. The authors have proposed an application of contra-rotating rotors and investigated its effectiveness for higher specific speed pump [3]. The most important feature of contra-rotating axial flow pump is that the rear rotor, installed instead of the stator of conventional type, plays a role not only to recover the static head but also to give the energy to the fluid directly, resulting in the reduction of the rotational speed and/or the pump size under the same specification of conventional axial flow pump [4]. It has been experimentally confirmed that the application of contra-rotating rotors results in better cavitation performance compared to the conventional one, which is favourable result for the higher specific pump. And also, because of the reduced head for each rotor, it is expected that the contra-rotating axial flow pump would have a stable operating characteristics, i.e. a negative slope of a head performance curve, over the flow rate range from the design to the shut off conditions. However, in our previous experiments [5], a positive slope of the head performance of the whole pump appeared in partial flow conditions, and this was found to come from the head performance of the rear rotor. Then it can be said that it is necessary to understand the internal flow behaviour in the rear rotor to establish the design guideline for the development of higher performance and more reliable contra-rotating axial flow pump.

In the present study, under this background, we carry out the experimental investigations of the internal flow filed of the rear rotor, especially at the partial flow rates, by Laser Doppler Velocimetry (LDV) for the main flow and the limiting streamlines observation using multi-coloured oil-film method [6,7] for the boundary layer flows on rotor surfaces. In addition, since the formation of the backflow near the hub surface at the exit of the front rotor was found to have a strong effect on the internal flow of the rear rotor, we also investigate the internal flow behaviour of the rear rotor by changing the rotational speed of the front rotor while keeping a dimensional flow rate being constant at the low value.

Received December 27, 2010; accepted for publication January 19, 2011: Review conducted by Prof. Zhongdong Qian. (Paper number O10062C)

Corresponding author: Satoshi Watanabe, Associate Professor, fmnabe@mech.kyushu-u.ac.jp 


\section{Experimental Apparatus and Measurement Procedure}

The test contra-rotating axial flow pump has been designed with the specified pump head of $H_{d}=4.0 \mathrm{~m}$ and the flow rate of $Q=70 \mathrm{~L} / \mathrm{s}$. By selecting the specific speed of each rotor as $1500\left[\mathrm{~min}^{-1}, \mathrm{~m}^{3} / \mathrm{min}, \mathrm{m}\right]$, the rotational speed was determined as $N_{f}=N_{r}=1225 \mathrm{~min}^{-1}$ so that both the front and the rear rotors have the same pump head of $2.0 \mathrm{~m}$. The each blade row was designed by using conventional empirical method [4], and the numbers of the blades are chosen as 4 and 5 for the front and the rear rotors respectively. Main dimensions of rotors are summarized in Table 1.

Table 1 Main dimensions of front and rear rotors

\begin{tabular}{|c|c|c|c|c|}
\hline \multirow{4}{*}{$\begin{array}{c}\text { Front } \\
\text { rotor }\end{array}$} & Hub & Mid-span & Tip \\
\cline { 2 - 5 } & Dlade number & $100 \mathrm{~mm}$ & $149 \mathrm{~mm}$ & $198 \mathrm{~mm}$ \\
\cline { 2 - 5 } & Blade profile & NACA4410 & \multicolumn{3}{|c|}{ NACA4408 } & NACA4406 \\
\cline { 2 - 5 } & Solidity & 1.29 & 0.90 & 0.70 \\
\cline { 2 - 5 } & Stagger angle & $51.72^{\circ}$ & $68.48^{\circ}$ & $75.85^{\circ}$ \\
\hline \multirow{3}{*}{$\begin{array}{c}\text { Rear } \\
\text { rotor }\end{array}$} & Blade number & \multicolumn{4}{|c|}{5} \\
\cline { 2 - 5 } & Blade profile & NACA4410 & NACA4408 & NACA4406 \\
\cline { 2 - 5 } & Solidity & 0.84 & 0.72 & 0.60 \\
\cline { 2 - 5 } & Stagger angle & $64.24^{\circ}$ & $72.54^{\circ}$ & $77.56^{\circ}$ \\
\hline
\end{tabular}

Figure 1 shows the sectional view of the test pump. The hub diameter is $100 \mathrm{~mm}$, the casing one is $200 \mathrm{~mm}$ and a blade tip clearance is $1.0 \mathrm{~mm}$. A set of two shafts, which are inner and outer ones, is required for each front and rear rotor, and each shaft is connected via a torque-meter to a respective motor. We can control the rotational speed of both rotors independently with the aid of the electric inverter installed to each motor.

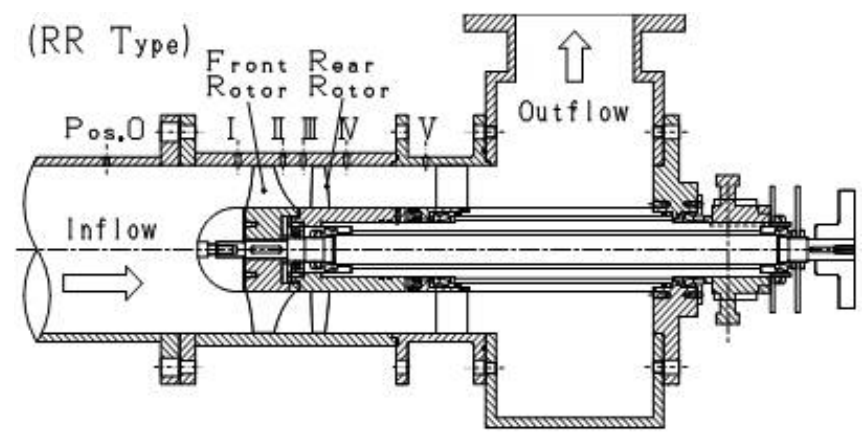

Fig. 1 Sectional view of test pump

For the pressure performance evaluation, the static head differences on the casing wall are measured between the up- and downstream sections of rotors, Pos.0, III and V in Fig. 1. Then, the head rises of the front and the rear rotors, $H_{f}$ and $H_{r}$, and the total pump head $H_{R R}=H_{f}+H_{r}$ are evaluated by adding the dynamic head difference of the sectional averaged axial velocity into the corresponding measured static head difference. Although we do not take account of the circumferential velocity component into the pump head evaluation, the qualitative discussion can be made for the tendency of the head performance curve. The flow rate $Q$ is obtained from an orifice meter installed far upstream of the pump. Then, the hydraulic efficiency of pump $\eta$ is calculated as the ratio of the water power to the shaft power after eliminating the mechanical losses which have been separately measured in advance.

The pump casing is made of acrylic transparent resin. Therefore, the internal flow measurement with Laser Doppler Velocimetry (LDV) is possible. As well known, the LDV basically measures the moving velocity of small particle which randomly passes through the intersectional point of two laser beams. Therefore, for the unsteady measurement, huge amount of particles are necessary to have continuously seeded particles at the intersectional point, i.e. at the measuring axial and radial location. However, since we are just interested in the averaged blade-to-blade velocity distributions, we use small amount of particles and employ the concept of ensemble averaging technique. In the measurement, we only use small impurities contained in the water as seeding particles, and carry out the LDV measurement for axial and circumferential components separately. For the each measured particle, we simultaneously detect the rotation angle of the one of front and rear rotors by the rotation counter with 120 pulses per one revolution. We obtain more than 4000 sets of each velocity component and phase angle of the corresponding rotor. By mapping the velocity data from time domain onto the phase angle domain, we can obtain the blade-to-blade velocity distributions at the measuring axial and radial location. The measurement basically can be done at arbitrary axial and radial positions, whereas the velocity near the casing and hub walls and blade surfaces cannot be measured because of the dispersion of the laser beam on the solid walls.

For the flow observation near the solid surfaces, the limiting streamlines on the rotating walls of flow passages are visualized by multi-colored oil-film method [6, 7]. Before the pump operation, well compounded green, red, blue colored oil are painted respectively on the pressure and suction surfaces of the blade and the hub surface. All colored oils are made of Petroleum hydrocarbons based lubricating oil as base solvent, color paint oil, Oleic acid for dispersions of paint oil, and grease for the 
adjustment of stickiness. After three minutes operation at the constant flow rate and constant rotational speed of front and rear rotors, we observe the limiting streamlines on rotor surfaces.

Figure 2 shows the pump characteristics of the test pump. The test pump yields pump head $H_{R R}=3.6 \mathrm{~m}$, which does not satisfy the designed head of $H_{d}=4 \mathrm{~m}$, and the maximum efficiency $\eta_{R R}=83 \%$ at the design point of $Q=70 \mathrm{~L} / \mathrm{s}$. As the feature of contrarotating type, the rear rotor head of $H_{r}$ is higher than the front rotor one $H_{f}$ at partial flow rates, which means more stable head characteristic curve with the negative slope of $d H / d Q<0$. However the positive slope appears in the flow rate range of $Q<28 \mathrm{~L} / \mathrm{s}$, and the corresponding rear rotor head also has the positive slope. Therefore, in the present study, in order to understand the internal flow structure of the rear rotor at partial flow rate, $Q=28 \mathrm{~L} / \mathrm{s}$, the detailed LDV measurement and the limiting streamlines observation by the oil-film method were carried out. The effect of the exiting flow of the front rotor on the internal flow field of the rear rotor is also examined by carefully decreasing the rotational speed of the front rotor while keeping the flow rate to eliminate the backflow near the hub surface appearing at the outlet of the front rotor.

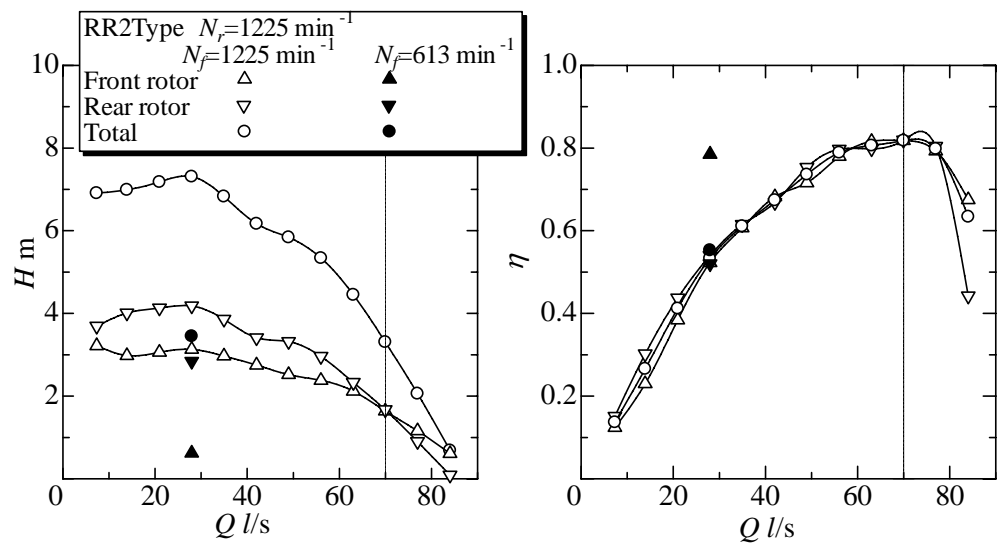

Fig. 2 Pump characteristics on head and efficiency

\section{LDV measurement}

Figure 3 shows the meridional streamlines drawn using the time-averaged axial velocity component obtained from LDV measurements at the partial flow rate of $Q=28 \mathrm{~L} / \mathrm{s}$. Broken lines depict the boundary between thru-flow and backflow regions where axial velocity components are zero. Around the front rotor, the backflow region can be found near the blade tip at the inlet as well as near the hub surface at the outlet, in the same manner to the conventional axial flow pump rotor. Although it is expected that the centrifugal force effect results in the stable head characteristics, i.e. the negative slope of $H_{f}-Q$ characteristics of the front rotor, only the gradual negative slope can be seen. It is considered that the head drop due to the blockage effect with the inlet backflow is stronger than the head rise due to the centrifugal force effect with the outlet backflow. Around the rear rotor, on the contrary, we can find only the inlet backflow near the blade tip and never find the backflow at the outlet. In the rear rotor, as we can easily imagine from the working principle, i.e. the velocity triangles of contra-rotating pump, the absolute circumferential velocity component becomes smaller at the exit and the resulting centrifugal force becomes smaller compared to that of the front rotor. This seems to be a reason why the backflow cannot form near the hub surface at the exit and the head drop occurs around this flow rate (Fig. 2.)

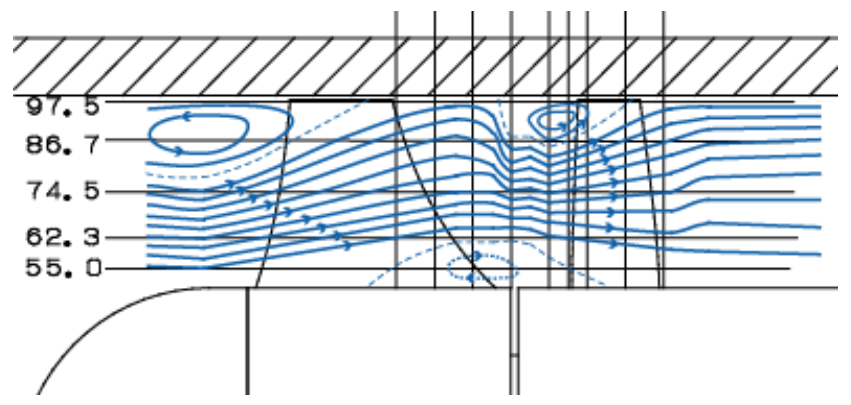

Fig. 3 Meridional streamlines at $Q=28 \mathrm{~L} / \mathrm{s}$

Figure 4 shows the blade-to-blade distributions of relative velocity vectors at the partial flow rate of $Q=28 \mathrm{~L} / \mathrm{s}$ at the radial positions of $r / R_{c}=0.975,0.623$ and 0.555 ( $R_{c}$ denotes a radius of the casing wall) measured by the LDV synchronized with the rear rotor rotation. The grey-colored region means the backflow region where the local axial velocity is negative. The corresponding distribution of the casing wall static head-rise from the front rotor inlet [8] is plotted in Fig. 5.

Looking at the velocity field near the blade tip in Fig. 4(a), we can see the huge backflow region with the strong swirl component at the inlet of the rear rotor. This backflow region appears not only near the leading edge but also at the every inlet region. The velocity there widely varies in the circumferential direction because of the flow turning at the leading edge, although the flow exiting from the front rotor is rather uniform. This backflow region is observed in the mid-passage, which is considered 
to lead the decrease of the blade loading near the tip, resulting in the head drop of the rear rotor. It is interesting to see that, despite that the backflow region is observed in the mid-passage, there is no backflow region near the suction surface. It is supposed that the fluid there is supplied from inner region due to the secondary flow, and then the flow goes along the suction surface of the blade. From casing wall pressure distribution in Fig. 5, the low static head region is widely observed in circumferential direction on the suction surface in spite of the increase of the static head given by the front rotor. At the partial flow rate, the large angle of attack results in the large flow turning around the leading edge from pressure to suction surfaces of the blade, leading the observed large low pressure region.

Perhaps due to the existence of the huge backflow region near the tip, the flow in the inner region (for instance at $r / R_{c}=0.623$ as shown in Fig. 4(b)) is almost the thru-flow except near the leading edge of the blade and the magnitude of the relative velocity is remarkably large. At $r / R_{c}=0.555$, the backflow region associated with the three-dimensional flow of the front rotor (see Fig. 3 ) can be seen in the gap between the front and rear rotors. Since the LDV measurement could not be done near the hub surface due to several regions, we will discuss about it using the results of limiting streamline visualization in the next section.

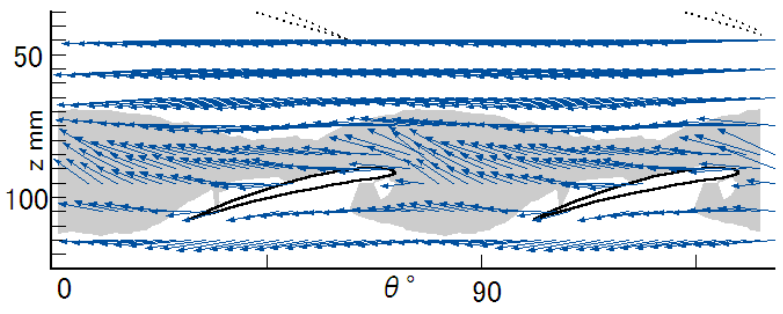

(a) $r / R_{c}=0.975$ section

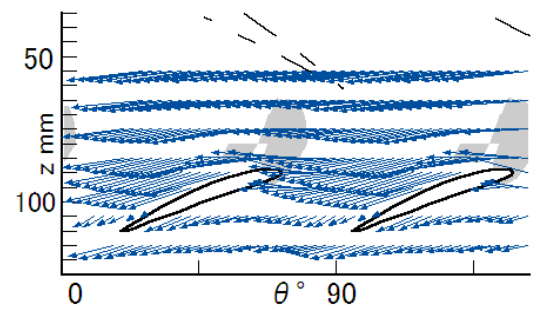

(b) $r / R_{c}=0.623$ section

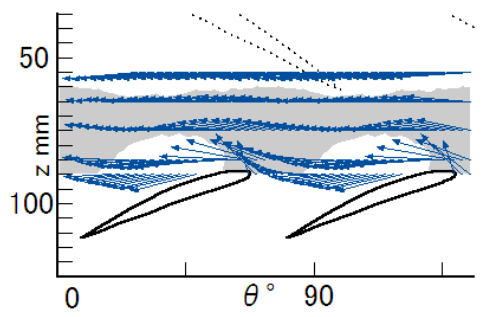

(c) $r / R_{c}=0.555$ section

Fig. 4 Velocity vector distribution of rear rotor at $Q=28 \mathrm{~L} / \mathrm{s}$

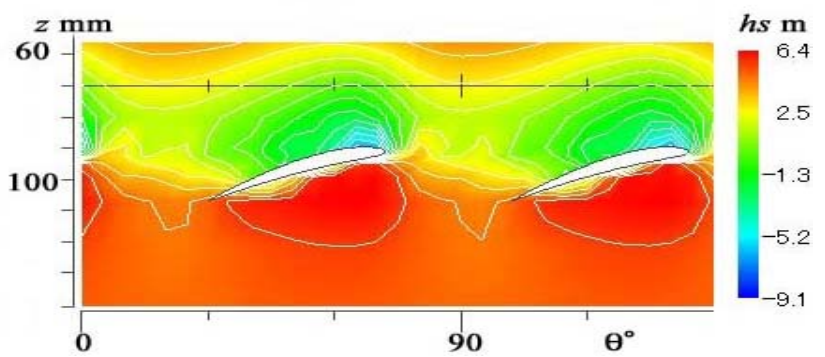

Fig. 5 Casing wall static head of rear rotor at $Q=28 \mathrm{~L} / \mathrm{s}$

\section{Limiting Streamline Visualization}

\subsection{Limiting Streamlines in Front Rotor}

In order to state clearly the differences of limiting streamlines between the front and rear rotors, we would like to firstly describe about those in the front rotor in this sub-section.

Figure 6 shows the visualized limiting streamlines on the suction (left hand side) and the pressure (right hand side) surfaces of the front rotor blade at the partial flow rate of $Q=28 \mathrm{~L} / \mathrm{s}$. On the suction surface of the blade, the low energy fluids inside the boundary layer developing on the hub surface go radially along the blade due to the secondary flow as indicated by the arrow (1). The same flow pattern can be seen on the pressure surface as depicted by (3), whereas the secondary flow seems to be stronger for the suction surface. These secondary flows are formed by the unbalance between the radial pressure gradient caused by the swirling main flow and the centrifugal force working on the low energy fluids inside the boundary layer; the boundary layer flow on rotor surfaces has larger swirling flow resulting in the larger centrifugal force than that in the main flow.
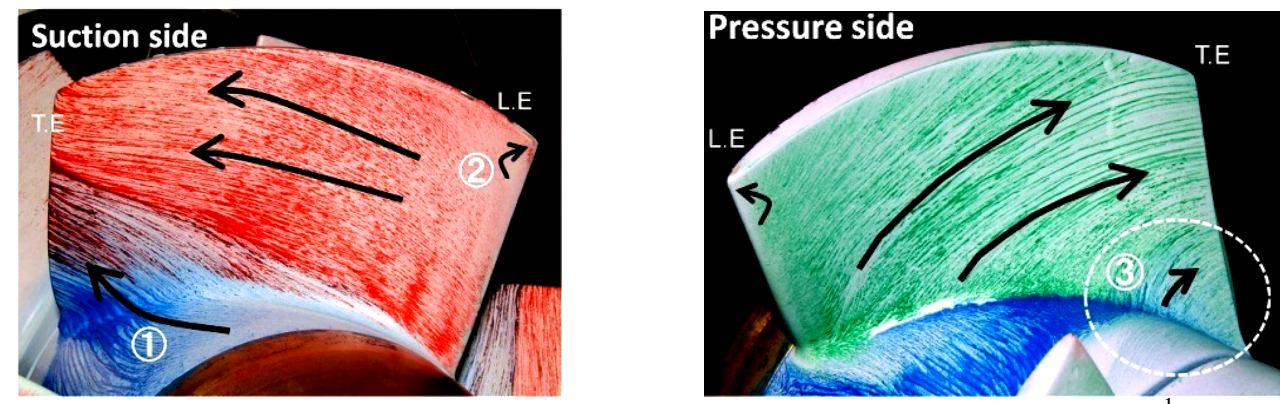

Fig. 6 Limiting streamlines of front rotor at $Q=28 \mathrm{~L} / \mathrm{s}, N_{f}=N_{r}=1225 \mathrm{~min}^{-1}$

As we can see later from Fig. 9(a) showing the limiting streamlines on the hub surface, the backflow develops on the hub surface at the exit of the front rotor and collides with the oncoming flow from the upstream in the blade passage, forming the flow separation line in the blade passage. After the collision, some part of the backflow flows around the trailing edge of the blade toward the pressure side as depicted by the arrow (9) shown in Fig. 9(a), and then flows radially along the pressure side as 
indicated by the arrow (3) shown in Fig. 6. On the other hand, at the inlet, we can find the flow exiting from the leading edge near the blade tip for both suction and pressure surfaces as depicted by the arrow (2), indicating the existence of the inlet backflow near the tip. The above observed limiting streamlines on the front rotor surfaces are resemble for those on the rotor surfaces of the conventional pump with rotor and stator [6].

\subsection{Limiting Streamlines in Rear Rotor}

Figure 7 shows the visualized limiting streamlines on the suction and the pressure surfaces of the rear rotor blade at the partial flow rate of $Q=28 \mathrm{~L} / \mathrm{s}$. On the suction surface of the blade, we can recognize the back flow near the leading edge of the blade tip as indicated by the arrow (4), which is similar to that observed in the front rotor (2) in Fig.6). As similar to the front rotor, the fluids in the boundary layer on the hub surface flow onto the suction surface as depicted by the arrow (5). We can see the greencolored region near the trailing edge on the suction surface (6), indicating the flow turns around the trailing edge from the pressure to the suction surfaces. This flow turning can be confirmed by the limiting streamlines on the hub surface, shown later in Fig. 9 (a). Since the backflow cannot be recognized in the blade-to-blade distribution of the velocity vectors measure by the LDV (Fig. 4), this behavior is considered to be limited near the blade surface. As shown later in Fig. 9(a), the limiting streamlines on the hub surface of the rear rotor are very complicated. The fluids in the hub boundary layer near the pressure surface exiting along the blade from the rear rotor go around the trailing edge and return to the blade passage. One part of those fluids reaches the rotor inlet and turns around the blade leading edge toward the suction surface of the blade as indicated by the dotted arrow (10) in Fig. 9(a). Another part encounters the oncoming boundary layer flow and turns toward the suction surface, while the rest flows downstream in parallel with the pressure surface of the adjacent blade. In the rear rotor, we never observe the flow separation lines on the hub surface, meaning that the back flow is limited just near the hub surface.
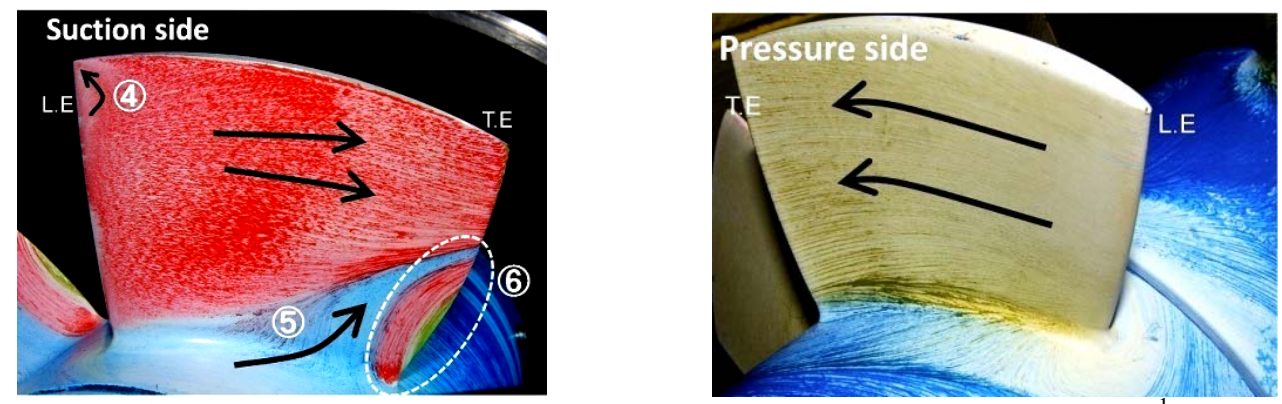

Fig. 7 Limiting streamlines of rear rotor at $Q=28 \mathrm{~L} / \mathrm{s}, N_{f}=N_{r}=1225 \mathrm{~min}^{-1}$

On the pressure surface, as shown in Fig. 7, the flow along the surface is parallel and no secondary flow can be seen. This is in contrast with that of the front rotor, where the flow from the boundary layer on the hub was observed also on the pressure side, due to the strong backflow developing at the outlet near the hub wall. This result supports the meridional flow observation shown in Fig. 3; the significant backflow cannot be observed on the hub surface at the outlet of the rear rotor, which might result in the reduction of the head rise of the rear rotor at the partial flow rates.

\section{Effect of Outlet Backflow of Front Rotor on Internal Flow of Rear Rotor}

The flow entering the rear rotor is very complicated due to the existence of the backflow of the front rotor. In order to examine the effect of the entering flow on the flow field in the rear rotor, we reduced the rotational speed of only the front rotor while keeping the flow rate. The rotational speed of the rear rotor is kept constant at the design value of $N_{r}=1225 \mathrm{~min}^{-1}$. By setting $N_{f}=613 \mathrm{~min}^{-1}$, the backflow at the exit of the front rotor vanished and the exiting flow from the front rotor becomes normal flow at the flow rate of $Q=28 \mathrm{~L} / \mathrm{s}$. Figure 8 shows the limiting streamlines on the suction and pressure surfaces of the rear rotor blade with $N_{f}=613 \mathrm{~min}^{-1}$. Figure 9 shows the limiting streamlines on the hub surfaces of the front and rear rotors with (a) $N_{f}=1225 \mathrm{~min}^{-1}$ and (b) $N_{f}=613 \mathrm{~min}^{-1}$. From the limiting streamlines on the hub surface of the front rotor, we can see that the flow entering the blade passage goes straightly through in the case with $N_{f}=613 \mathrm{~min}^{-1}$ as depicted by (11), while the backflow appears at the outlet and the separation line between the thru-flow and the backflow is formed in the case with $N_{f}=1225 \mathrm{~min}^{-1}$, indicating that the back flow at the outlet of the front rotor diminishes by reducing the rotational speed of the front rotor as we expected.
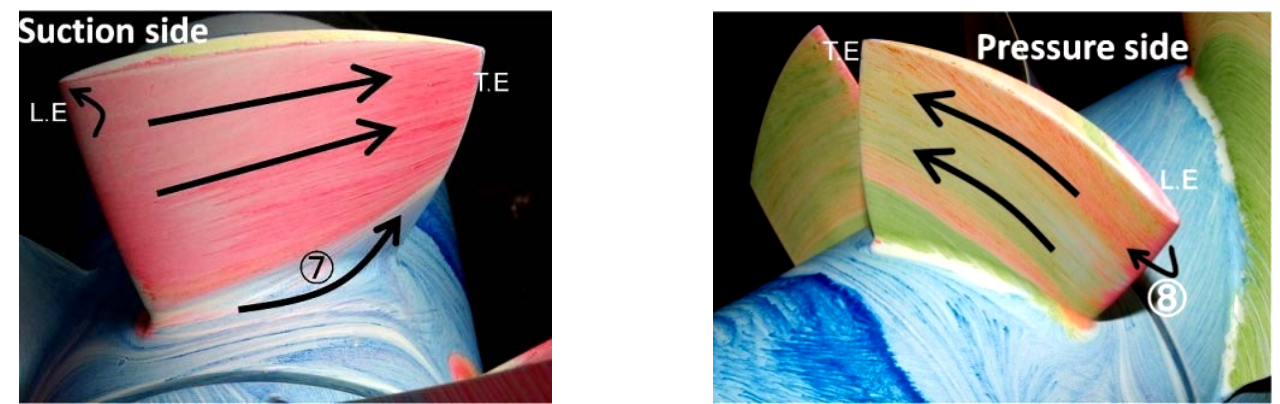

Fig. 8 Effect of rotational speed of front rotor on limiting streamlines on hub surface at $Q=28 \mathrm{~L} / \mathrm{s}, N_{f}=613 \mathrm{~min}^{-1}$ 


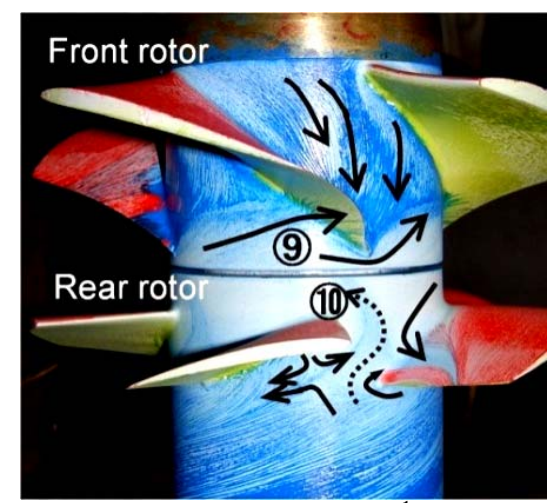

(a) $N_{f}=1225 \mathrm{~min}^{-1}$

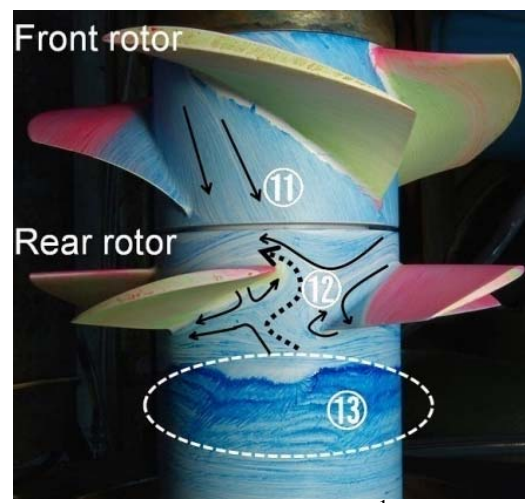

(b) $N_{f}=613 \mathrm{~min}^{-1}$

Fig. 9 Effect of rotational speed of front rotor on limiting streamlines on hub surface at $Q=28 \mathrm{~L} / \mathrm{s}$

Looking at the limiting streamlines of the rear rotor, even though the oncoming flow from the front rotor is drastically changed by reducing the rotational speed of the rear rotor, the flow pattern on the suction surface of the blade is somewhat similar to that with $N_{f}=613 \mathrm{~min}^{-1}$; we can see the backflow near the blade tip at the inlet as well as the radially outward flow from the boundary layer on the hub surface as depicted by the arrow (7). However, for $N_{f}=613 \mathrm{~min}^{-1}$, we cannot find the flow turning around the trailing edge of the blade, which is clearly observed for $N_{f}=1225 \mathrm{~min}^{-1}$ [ (6) in Fig. 7]. On the other hand, the limiting streamlines on the pressure surface can be seen to be significantly changed by reducing the rotational speed of the front rotor; as depicted by (8), the flow turns around the leading edge from the suction to the pressure surfaces, meaning that the stagnation point locates on the suction surface of the blade. This occurs because, by reducing the rotational speed of the front rotor, the circumferential velocity component of the flow exiting from the front rotor decreases resulting in the negative incidence angle at the inlet of the rear rotor, Nevertheless, the overall streamlines are slightly inclined in the radial direction indicating the effect of the secondary flow.

From the limiting streamlines on the hub surface of the rear rotor, the backflow is limited in the blade passage in the case with $N_{f}=1225 \mathrm{~min}^{-1}$ [Fig. 9(a)] and it reaches the inlet of the rear rotor and seems to turn around the leading edge of the blade toward the adjacent blade passage as depicted by the arrow (10. On the other hand, in the case with $N_{f}=613 \mathrm{~min}^{-1}$ [Fig. 9(b)], the similar flow pattern (12) can be seen in the blade passage, whereas the stagnant flow region can be found downstream of the rear rotor as depicted by (13. By reducing the rotational speed of the front rotor, the oncoming flow to the rear rotor becomes more or less uniform without the backflow on the hub surface, and then the effect of the centrifugal force of the main flow in the blade passage of the rear rotor becomes more apparent as evidenced by the appeared secondary flow on the pressure and suction surfaces of the rear rotor blade, which has been shown in Fig. 8. This indicates stronger radially toward three-dimensional flow which is considered to induce the stagnant flow region downstream of the rear rotor. In order to examine the validity of this hypothesis, we operate only the rear rotor without the front rotor as for the extreme case without the inlet swirl to the rear rotor. Figure 10 shows the limiting streamlines on the hub surface of the rear rotor, operated without the front rotor at $Q=28 \mathrm{~L} / \mathrm{s}$ with $N_{r}=1225 \mathrm{~min}^{-1}$. We can see the flow turning at the trailing edge from the pressure to the suction surfaces of the blade (14) as well as the large scale back flow (15) at the outlet. This flow pattern is somewhat similar to that in the front rotor shown in Fig. 9(a). We believe that the stagnant flow region (13) in Fig. 9(b) becomes back flow (15) in Fig. 10 (b), by removing the inlet swirl.

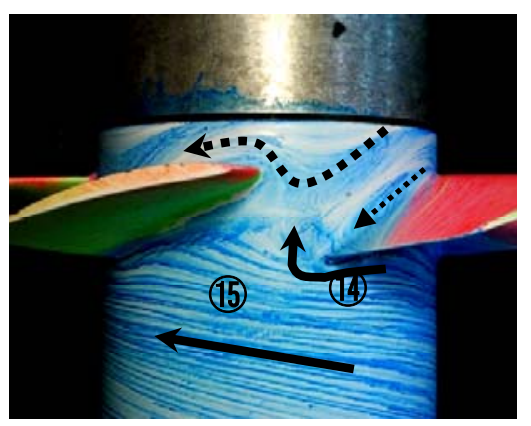

Fig. 10 Limiting streamlines of rear rotor operated without front rotor at $Q=28 \mathrm{~L} / \mathrm{s}$

Figure 11 shows the radial distributions of (a) axial and (b) circumferential components of absolute velocity measured by 5 hole yaw-meter at just upstream of the front rotor (Pos. I) and at just upstream of the rear rotor (Pos. III, downstream of the front rotor). Circumferential direction $\theta$ is defined in the direction of the rotation of the front rotor, and then the negative value of $V_{\theta}$ means that the swirling direction is that of the rear rotor rotation. Comparing the velocity distributions for the rotational speed of the front rotor of $N_{f}=1225 \mathrm{~min}^{-1}$ between upstream of front and rear rotors (open triangles for Pos. I and open circles for Pos. III), we can see the significant difference. However, by reducing the rotational speed of the front rotor, the velocity distribution upstream of the rear rotor (closed circles) becomes closer to those upstream of the front rotor (open triangles); the back flow can be found near the blade tip for the both cases , and the tangential velocity distributions are almost symmetry around $V_{\theta}=0$. It is 
supposed that, because the oncoming flows to the front rotor with $N_{f}=1225 \mathrm{~min}^{-1}$ and to the rear rotor with $N_{f}=613 \mathrm{~min}-1$ are similar, the limiting streamlines become slightly closer.

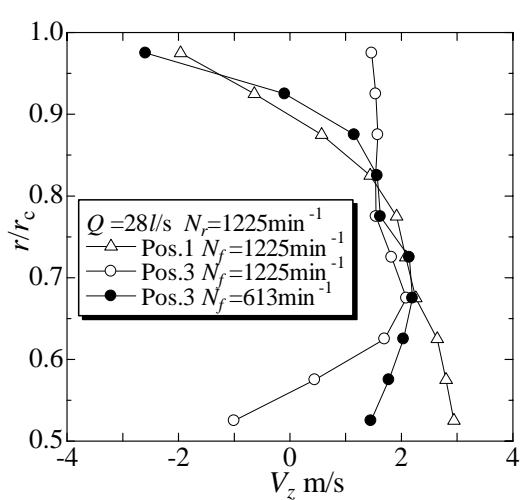

(a) $V_{z}$

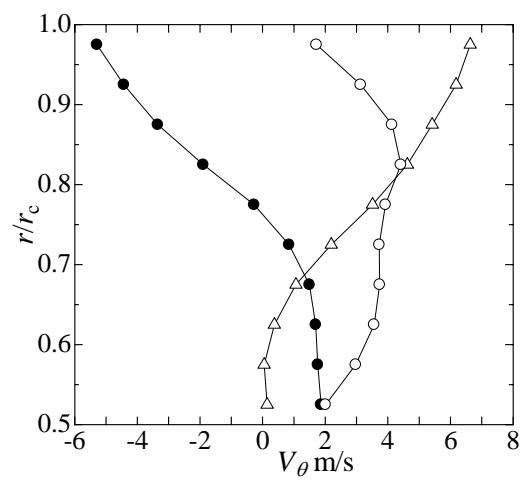

(b) $V_{\theta}$

Fig. 11 Comparison with the flow field of inlet of front rotor and rear rotor

We have plotted the pump performance with the reduced rotational speed of the front rotor, $N_{f}=613$ min ${ }^{-1}$ in Fig. 2 . By reducing the rotational speed of the front rotor, total efficiency is increased by $2 \%$. The efficiency of the front rotor is drastically increased since the corresponding flow coefficient approaches the design flow coefficient by reducing the rotational speed. The head rise of the front rotor is decreased with the increase of the flow coefficient and the decrease of the rotational speed. On the other hand, the efficiency of the rear rotor does not change, despite that the internal flow of the rear rotor is significantly changed. Then, it can be said that the increase of the efficiency with the decrease of the head rise of the front rotor decreases the shaft power consumed by the front rotor, which contributes the increase of the total efficiency of $2 \%$. This fact suggests that the rotational speed control of contra-rotating rotors has a beneficial effect on the improvements of pump performances.

\section{Conclusion}

In combination with the LDV measurements of internal flow and the limiting streamline observations, we have made clear the internal flow structure of the contra-rotating axial flow pump rotors operated at the partial flow rate. The effect of the exiting flow from the front rotor on the internal flow or rear rotor is also investigated. Main results can be summarized as follows.

1) The inlet back flow of the rear rotor elongates deeply into the blade passage, which seems to be the main cause of the head drop of the rear rotor at the partial flow rate.

2) Even though the tested flow rate is very low (40\% of design flow rate), the backflow did not develop on the hub surface at the outlet of the rear rotor. In general, the conventional axial flow pump suffers from the inlet backflow near the blade tip and the outlet backflow on the hub surface at such low flow rate, resulting in the radially toward three-dimensional flow inside the blade passage and the increase of the head rise due to the centrifugal force effect. The lack of the formation of the outlet backflow on the hub surface of the rear rotor seems to be responsible for the low head rise of the rear rotor in partial flow conditions.

3) The existence of the outlet backflow of the front rotor has a remarkable effect on the internal flow of the rear rotor, especially for the formation of the outlet backflow of the rear rotor. This implies that we can achieve the performance improvement of the contra-rotating pumps by the appropriate rotational speed controls of the contra-rotating rotors.

\section{Nomenclature}

$H \quad$ Head rise $[\mathrm{m}]$

$h_{s} \quad$ Casing wall static head rise from the inlet [m]

$N \quad$ Rotational speed of rotors $\left[\mathrm{mim}^{-1}\right]$

$Q \quad$ Flow rate $[\mathrm{L} / \mathrm{s}]$

$R_{c} \quad$ Casing radius [mm]

$r \quad$ Radius [mm]

$V_{z} \quad$ Axial component of absolute velocity [m/s]

$V_{\theta} \quad$ Circumferential component of absolute velocity [m/s]
Z Axial location from the leading edge of the front rotor blade [mm]

$\eta \quad$ Efficiency [-]

$\theta \quad$ Circumferential angle

\section{Subscript}

$f, r \quad$ Front and rear rotors

\section{References}

[1] Wada, A. and Uchida, S., 1999, “Improvement of Performance for Higher Specific Speed Axial-Flow Pump,” Torishima Review, (in Japanese), Vol. 13, pp. 32-35.

[2] White, J. W., Purnell, J. G. and Stricker, J. G., 1993, “In-Line Submersible Pump,” ASME, FED Summer Meeting, Paper No. 246, pp. 1-8.

[3] Furukawa, A., Cao, Y., Okuma, K. and Watanabe, S., 2000, “Experimental Study of Pump Characteristics of Contra-Rotating Axial Flow Pump,” the 2nd International Symposium on Fluid Machinery and Fluid Engineering, pp. 245-252.

[4] Furukawa, A., Shigemitsu, T. and Watanabe, S., 2007, "Performance Test and Flow Measurement on Contra-Rotating Axial Flow Pump,” Journal of Thermal Science, Vol. 16, No. 1, pp. 7-13. 
[5] Shigemitsu, T., Fukuyama, T., Furukawa, A., Okuma, K. and Watanabe, S., 2006, "Flow Measurement with LDV in ContraRotating Axial Flow Pump,” 23rd IAHR Symposium on Hydraulic Machinery and Systems, No. 045, pp. 1-10.

[6] Goto, A., Zangeneh, M. and Takemura, T., 1996, "Suppression of Secondary Flows in a Mixed Flow Pump Impeller by Application of 3-D Inverse Design Method; Part2 - Experimental Validation,” ASME Journal of Turbomachinery, Vol. 118, pp. 544-551.

[7] Furukawa, A., Usami, S., Tsunenari, Y., Watanabe, S. and Okuma, K., 2008, "Limiting Streamlines Measurement in ContraRotating Axial Flow Pump,” the 4th International Symposium on Fluid Machinery and Fluid Engineering, No. 4ISFMFE-IL20, pp. 1-6.

[8] Furukawa, A., Takano, T., Shigemitsu, T., Okuma, K. and Watanabe, S., 2006, "Blade Rows Interaction of Contra-Rotating Axial Flow Pump in Pressure Field on Casing Wall,” JSME International Journal, Ser. B, Vol. 49, No. 3, pp. $670-677$. 